\title{
Niobium doped BGO glasses: Physical, Thermal and Optical Properties
}

\author{
Khair-u-Nisa ${ }^{1}$, Ejaz Ahmed ${ }^{2}$, M. Ashraf Chaudhry ${ }^{3}$ \\ ${ }^{1,2,3}$ Department of Physics, Bahauddin Zakariya University, Multan (60800), Pakistan.
}

\begin{abstract}
IR- transparent niobium substituted heavy metal oxide glass system in formula composition (100-x) BGO75- $x \mathrm{Nb}_{2} \mathrm{O}_{5} ; 5 \leq x \leq 25$ was fabricated by quenching and press molding technique. Glassy state was confirmed by XRD. Density $\rho_{\text {exp }}$ varied $6.381 \mathrm{~g} / \mathrm{cc}-7.028 \mathrm{~g} / \mathrm{cc} \pm 0.06 \%$. Modifying behavior of $\mathrm{Nb}_{2} \mathrm{O}_{5}$ was corroborated by rate of increase in theoretical volume $V_{t h}$, measured volume $V_{\text {exp }}$ and oxygen molar volume $V_{M O^{-2}} . \mathrm{Nb}^{5+}$ had larger cation radius and greater polarizing strength as compared to $\mathrm{Ge} e^{4+}$ ions. It replaced $\mathrm{Ge}^{4+}$ sites introducing more NBOs in the network. Transformation temperatures $T_{g}, T_{x}$ and $T_{p 1}$ were $456-469^{\circ} \mathrm{C}$ $\pm 2{ }^{\circ} \mathrm{C}, 516-537{ }^{\circ} \mathrm{C} \pm 2{ }^{\circ} \mathrm{C}$ and $589-624^{\circ} \mathrm{C} \pm 2{ }^{\circ} \mathrm{C}$ respectively. In the range from room temperature to $400{ }^{\circ} \mathrm{C}$ the coefficient of linear thermal expansion $\alpha$ was $5.431 \pm 0.001 * 10^{-6} K^{-1}$ to $7.333 \pm 0.001 * 10^{-6} K^{-1} . \Delta T=T_{x}-T_{g}$ and $\Delta T_{P I}=T_{P I}-T_{g}$ varied collinearly with increase in niobium concentration and revealed thermal stability against devitrification. The direct bandgap $E_{g}$ values lay in $3.24-2.63 \mathrm{eV} \pm 0.01 \mathrm{eV}$ range and decreased due to impurity states of $\mathrm{Nb}^{5+}$ within the forbidden band. Mobility edges obeyed Urbach law verifying amorphousness of the compositions. The IR-transparency window extended from 0.38-6.1 $\mu \mathrm{m}$ in MIR which is wider than binary BGO glass as well as $B G O$ single crystal.
\end{abstract}

Keywords: BGO75; band gap; IR-transparent; Thermal stability; Urbach law

\section{Introduction}

Glass is an optically isotropic medium. It is an amorphous material with transparency window engineered via flexibility of composition. The unique class is oxide glasses. Their chemo-physical characteristics develop due to varying covalent, ionic or mixed covalent-ionic bonding nature of oxygen net within the cationic environment set up by the ratio of the cations [1]. There exists a great interest in ceramics fabricated via glass route. These transparent or translucent ceramics are clusters of more or less ordered nano/micro motifs embedded in amorphous matrix [2]. Transparent glasses on ceramization at the expense of some transparency may inject and multitude unique photonic, electro-optic, piezo-optic and/or magneto-optic properties.

$\mathrm{Bi}_{3} \mathrm{Ge}_{4} \mathrm{O}_{12}$ (BGO) are synthetic eulytine crystals. Fabrication of BGO single crystal is possible only with highly pure oxides and exact stichometric control of composition and thermal history [3]. It is of great importance in photonic materials and has diverse technological applications [2-6]. The binary system $\mathrm{Bi}_{2} \mathrm{O}_{3}-$ $\mathrm{GeO}_{2}$ exhibits glassy state in limited range of composition in $\mathrm{GeO}_{2}$ rich part of composition [4]. BGO glass ceramics are relatively easy to fabricate and cost effective [7]. These ceramics due to their technologically important features like IR-transmission, luminosity around $1.5 \mu \mathrm{m}$ and non-linear properties can replace BGO single crystals [8]. It is of great technical interest to probe the $\mathrm{Bi}_{2} \mathrm{O}_{3}-\mathrm{GeO}_{2}$ system with third oxide like $\mathrm{Nb}_{2} \mathrm{O}_{5}$ which could enhance its glass formation boundaries, foster its optical response in MIR and may provide control over ceramization of BGO crystallites [9].

The objective of the present study is to report the glass formation in $(100-x)\left[2.5 \mathrm{Bi}_{2} \mathrm{O}_{3}-7.5 \mathrm{GeO}_{2}\right.$ (BGO75)] $-x \mathrm{Nb}_{2} \mathrm{O}_{5} ; 5 \leq x \leq 25$ system with $\mathrm{Nb}_{2} \mathrm{O}_{5}$ replacement and lit upon the physical and opto-thermal properties of the ternary system. In this system $\mathrm{GeO}_{2}$ is a classical network former, $\mathrm{Bi}_{2} \mathrm{O}_{3}$ a conditional glass former and $\mathrm{Nb}_{2} \mathrm{O}_{5}$ is incorporated as transition metal dopant that could bring about thermo-mechanical stability and network reinforcement extending the transmission window [7]. Such a study is of academic as well as technical interest as BGO eulytine structure single crystal and binary BGO ceramics are being explored extensively due to their opto-electronic applications for WDM and radiation detectors/absorbers. In authors' knowledge ab-initio study on ternary glass system niobium doped BGO75 glasses is reported for the first time.

\subsection{Sample Fabrication}

\section{Experimental Procedures}

Glass samples (10g each) of nominal composition (100-x) (BGO75) $-x \mathrm{Nb}_{2} \mathrm{O}_{5} ; 5 \leq x \leq 25$ in mole $\%$ ratio of oxides were prepared by melt-quench technique. Analytical grade oxides (Sigma Aldrich: $99.9 \%$ or 99.99\% purity; with trace metal basis) were used. Powders were mixed thoroughly in a quartz mortar and pestle for half an hour. Admixture was put in platinum crucible and dried in box-type furnace for one hour at $400^{\circ} \mathrm{C}$ in order to remove moisture. Then the crucible was shifted to another open air pre-heated furnace at temperature 
$1323 \mathrm{~K}$ to $1443 \mathrm{~K}$ with varying composition of the sample. Fused was homogenized for 20-30 min. Circular sample disks were casted using a preheated stainless steel mold which was pre-annealed for one hour at $200{ }^{\circ} \mathrm{C}$ to avoid thermal shock due to large temperature difference of melt and mold. Fabricated samples were annealed at $350{ }^{\circ} \mathrm{C}$ for one hour and furnace cooled to room temperature. Glasses varied in yellow shade to brownish tint as the niobium concentration and soaking temperature/time was increased. Disk samples were wet polished mechanically to optical finish using carbide papers of grit size 400-2000 gradually. Samples withstood mechanical stability while polishing and grinding.

\subsection{X-ray Diffraction (XRD)}

XRD pattern of the fabricated samples were measured using Geiger flex D/Max-3D M/S Rigaku Corporation, Japan. It exploited nickel filter and copper target. CuK $\alpha$ radiation with $\lambda=1.542 \mathrm{~nm}$ was used at power rating $35 \mathrm{kV} \times 30 \mathrm{~mA}$. XRD pattern was scanned in the range $5^{\circ}$ to $80^{\circ}$ of $2 \theta$ with a step size of $0.05^{\circ}$. Plano-parallel disks were used for XRD measurements.

\subsection{Density}

Density of the bead samples was measured exploiting Archimedes' buoyant liquid method using Idoethane (density1.595 g/cc) as immersion liquid with single pan electronic balance of accuracy $\pm 0.00001 \mathrm{~g}$. Error in density value was less than $\pm 0.06 \%$ and results were reproducible to $\pm 0.01 \%$. Density was calculated using the relation

$$
d_{\text {exp }}=\frac{m_{1}}{\left(m_{1}+m_{2}\right)-m_{3}} * d_{\text {liq }}
$$

Where $m_{1}$ is the mass of the sample in air, $m_{2}$ is the mass of the pan in liquid, $m_{3}$ is the mass of pan and sample in the liquid and $d_{l i q}$ the density of the immersion liquid used. The difference $\left(m_{1}+m_{2}\right)-m_{3}$ gives mass of the equivalent volume of liquid displaced. Density values were confirmed by comparing the values obtained by automated ULTRAPYCNOMETER 1000 from Quantachrome Instruments with result accuracy $\pm 0.03 \%$ and repeatability $\pm 0.01 \%$. The average volume of each sample immersed in argon purged cell was measured for three preprogrammed runs. The volume standard deviation was less than $0.0035 \%$. The temperature of the argon purged cell was $26^{\circ} \mathrm{C} \pm 1^{\circ} \mathrm{C}$ during the measurement.

\subsection{Dilatometry}

NETZSCH dilatometer 402E was used to record co-efficient of linear thermal expansion (CTE). Sample powder was heated at the nominal rate $5{ }^{\circ} \mathrm{C} / \mathrm{min}$ within an alumina crucible from room temperature to $400{ }^{\circ} \mathrm{C}$. CTE $(\alpha)$ was noted from the slope of $\frac{d L}{L_{o}}$ vs. temperature curve through computer interfacing using the relation

\subsection{Characteristic Temperatures}

$$
\alpha=\frac{1}{d T}\left(\frac{d L}{L_{o}}\right)
$$

DTA-curves of the sample in the range $25-850{ }^{\circ} \mathrm{C}$ were recorded on STA-PT-1600, Linseis, Germany under static environment. Micro powder sample was heated at nominal rate of $10^{\circ} \mathrm{C} / \mathrm{min}$. in alumina crucible. $T_{g}$ the glass transition temperature, $T_{x}$ the onset of crystallization temperature, $T_{p i}$ for $i^{\text {th }}$ crystallization peak temperature were noted from DTA curves. The glass transition temperature $T_{g}$ denotes the temperature range of an endothermic shift of baseline in the DTA curve. The crystallization onset $T_{x}$ refers to temperature where exothermic inflection just begins. The temperature of exothermic peak inflection $T_{p}$ corresponds to maximum crystallization rate. Liquidus temperature $T_{l}$ is the minimum of endothermic peak apex value in the DTA-curve.

\subsection{UV-VIS-NIR spectra}

Optically finished samples (disks) were used to record UV-VIS-NIR spectra of the samples exploiting spectrophotometer U-4000, Hitachi with resolution $=2 \mathrm{~nm}$ in $200-2500 \mathrm{~nm}$ range. Absorbance A and percent transmission $T \%$ were recorded independently applying baseline correction and computer interface.

\subsection{FTIR spectra}

FTIR spectra of some annealed parallel disk samples of thickness 1-2 mm were recorded on model Shimadzu 8400s at room temperature. The recorded energy range was $400-4000 \mathrm{~cm}^{-1}$ with resolution exploited to be $2 \mathrm{~cm}^{-1}$. An average of 20 runs was recorded.

\subsection{Composition}

\section{Results and Discussion}

Glasses in the system $(100-x)\left[2.5 \mathrm{Bi}_{2} \mathrm{O}_{3}-7.5 \mathrm{GeO}_{2}(\mathrm{BGO} 75)\right]-x \mathrm{Nb}_{2} \mathrm{O}_{5}$ with $5 \leq x \leq 25$ in mole $\%$ ratio were fabricated. The network former oxide $\mathrm{GeO}_{2}$ and conditional glass former $\mathrm{Bi}_{2} \mathrm{O}_{3}$ had fixed proportion 
75:25. This ratio lay within the glass forming $\mathrm{GeO}_{2}$ rich region of the binary $\mathrm{Bi}_{2} \mathrm{O}_{3}-\mathrm{GeO}_{2}$ phase diagram [4]. $2 \mathrm{~d}$ transition metal niobium penta oxide $\left(\mathrm{Nb}_{2} \mathrm{O}_{5}\right)$ replaced the binary in gradual steps of $5 \mathrm{~mol} \%$. The glasses were named accordingly BGO75-Nbx as reported in Table1 with the mol $\%$ ratio of $\mathrm{Nb}_{2} \mathrm{O}_{5}$ in each glass.

\subsection{XRD}

Fig.1 shows X-ray diffraction pattern of five niobium doped BGO samples. All compositions exhibited a broad diffraction envelope. This confirms the amorphous state of the samples in BGO75-Nb glasses.

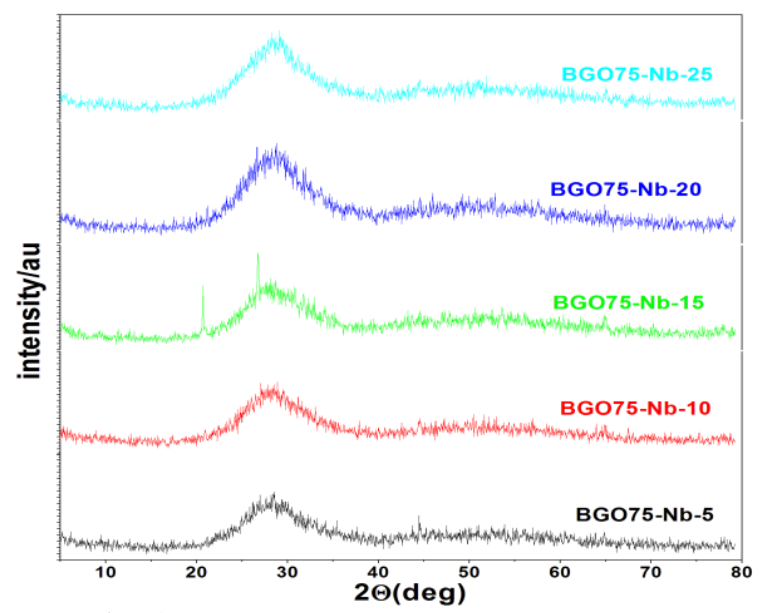

Fig. 1 XRD-pattern of BGO75-Nb glasses

\subsection{Density and Molar volume}

The simplest way of detecting structural changes in glass network, with compositional variation is by probing its rigidness/flexibility by means of density, molar volume and/or oxygen molar volume of the glass system. Theoretically density is a linear function of composition and can be estimated additively for glass as $\rho_{t h}=\frac{\sum x_{i} \rho_{i}}{\sum n_{i}}$; where $\rho_{i}$ is the density of $i^{t h}$ oxide component and $x_{i}$ is its mole fraction in the composition. Molar volume $V_{M}$ is calculated by dividing the molar mass of the composition by its density as $V_{M}=\frac{\sum x_{i} m_{i}}{d}$ and oxygen molar volume $V_{M O}{ }^{-2}$ of the network of oxygen in one mole of glass composition can be calculated using the relation $V_{M O^{-2}}=V_{M} * \frac{1}{\sum x_{i} n_{i}}$; where $n_{i}$ denotes number of oxygen atoms contributed by each oxide [7]. It is volume fraction occupied by anions in the network.

In Table1 are reported the experimentally measured and theoretically estimated densities $\rho_{e x p}, \rho_{t h}$ and corresponding molar volume $V_{M}$ exp,$V_{M}$ th along with oxygen molar volume $V_{M O^{-2}}$. Values obtained by using pyknometric measurement $\rho_{p y k}$ and $V_{M p y k}$ are also included in Table1.

Table1Glass code, mol\% concentration, densities calculated theoretically $\rho_{t h}$, measured by Archimedes' principle $\rho_{e x p}$, using pycnometer $\rho_{p y k}$, corresponding molar volumes $V_{t h}, V_{\text {exp }}, V_{p y k}$ and oxygen

\begin{tabular}{ccccccccc}
\multicolumn{1}{c}{$\begin{array}{c}\text { molar volume } V_{m O} \\
\text { code }\end{array}$} & $\begin{array}{c}\mathbf{N b}_{\mathbf{2}} \mathbf{O}_{\mathbf{5}} \\
\mathrm{mol} \%\end{array}$ & $\begin{array}{c}\mathbf{d}_{\text {Exp }} \\
\mathrm{g} / \mathrm{cc}\end{array}$ & $\begin{array}{c}\mathbf{V}_{\text {exp }} \\
\mathrm{cc}\end{array}$ & $\begin{array}{c}\mathbf{d}_{\text {th }} \\
\mathrm{g} / \mathrm{cc}\end{array}$ & $\begin{array}{c}\mathbf{V}_{\text {th }} \\
\mathrm{cc}\end{array}$ & $\begin{array}{c}\mathbf{d}_{\text {Pyk }} \\
\mathrm{g} / \mathrm{cc}\end{array}$ & $\begin{array}{c}\mathbf{V}_{\text {pyk }} \\
\mathrm{cc}\end{array}$ & $\begin{array}{c}\mathbf{V}_{\mathbf{m o}}{ }^{-2} \\
\mathrm{cc}\end{array}$ \\
\hline BGO75-Nb05 & 0.52 & 7.028 & 27.73 & 5.39 & 36.15 & 8.4278 & 23.13 & 15.324 \\
BGO75-Nb10 & 1.10 & 6.384 & 30.53 & 5.39 & 36.17 & 5.9908 & 32.53 & 17.826 \\
BGO75-Nb15 & 1.73 & 6.469 & 30.11 & 5.38 & 36.2 & 6.3409 & 30.72 & 18.458 \\
BGO75-Nb20 & 2.44 & 6.381 & 30.52 & 5.38 & 36.22 & 6.3766 & 30.54 & 19.688 \\
BGO75-Nb25 & 3.23 & 6.384 & 30.50 & 5.37 & 36.25 & 6.0779 & 32.03 & 20.765 \\
\hline
\end{tabular}

Fig.2 presents the results in graphical form as a function of increasing niobium-oxide concentration in mol\%. Theoretically the density of the glasses decreased from $5.39 \mathrm{~g} / \mathrm{cc}$ to $5.37 \mathrm{~g} / \mathrm{cc}$ with increasing mol\% concentration of $\mathrm{Nb}_{2} \mathrm{O}_{5}$. It was expected as lighter mass cations replaced the relatively heavier average cation in the BGO75 glass. But experimentally this regular monotonic decrease was not observed. The density values exploiting liquid displacement method $\rho_{\text {exp }}$ varied $6.381 \mathrm{~g} / \mathrm{cc}-7.028 \mathrm{~g} / \mathrm{cc} \pm 0.06 \%$ whereas density measured using pycnometer was $5.9908 \mathrm{~g} / \mathrm{cc}-8.4278 \mathrm{~g} / \mathrm{cc} \pm 0.03 \%$. It can be noted that the densities measured using two different methods are strongly correlated and comparable in magnitude but higher than those estimated through 
formula composition. The difference in two measured values of densities may be due to rounding off the mass values of samples to four significant figures for Pyknometric volume measurements.

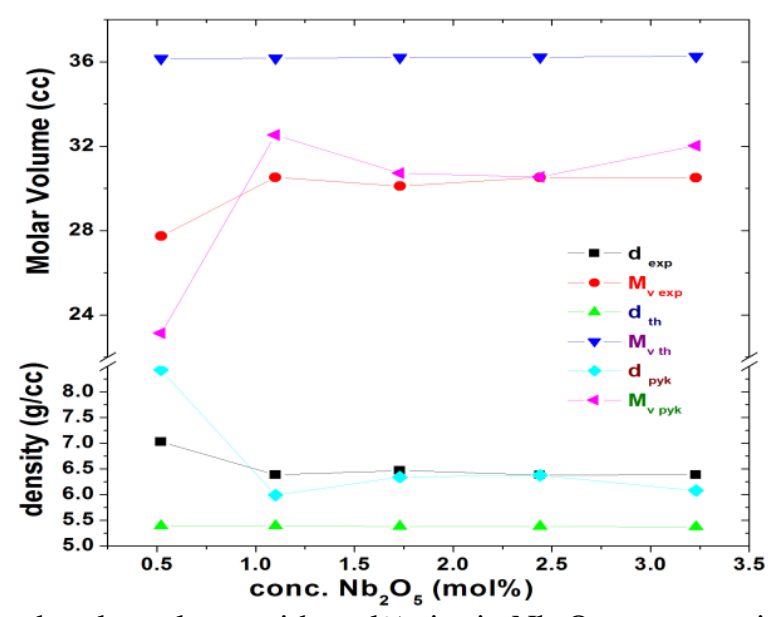

Fig. 2 Variation in density and molar volume with $\mathrm{mol} \%$ rise in $\mathrm{Nb}_{2} \mathrm{O}_{5}$ concentration for $\mathrm{BGO} 75$ glasses

The density values show that the glass becomes more rigid and compact as niobium is introduced in the composition. This may be due to the fact that ionic radii of cations are in the order $\mathrm{Bi}^{3+}>\mathrm{Nb}^{5+}>\mathrm{Ge}^{4+}[10]$. Also the polarizing strength of niobium ion is less than that of bismuth ion but greater than $\mathrm{Ge}^{4+}[10,11]$. These factors may result in change in coordination polyhedra of the network. $\mathrm{Nb}^{5+}$ ion can occupy some positions within the network as modifier replacing $\mathrm{Ge}^{4+}$ atom or ion exchange with $\mathrm{Bi}^{3+}$ ions. Resulting increase in molar volume occupied is an indication that it replaces Ge sites. Logically, molar volume increases with (i) the no. of oxygen atoms (ii) concentration and radius of cation (iii) rise in co-ordination number (iv) decrease in polarizability of cation [12]. These factors collectively exhibited monotonic increase in volume. Molar volume and Oxygen molar volume both increased with increasing mol\% concentration of $\mathrm{Nb}_{2} \mathrm{O}_{5}$ initially up to $\mathrm{Nb} 10$. This implied modifying behavior of $\mathrm{Nb}^{5+}$ ions incorporating NBOs and depolymerization in the system.

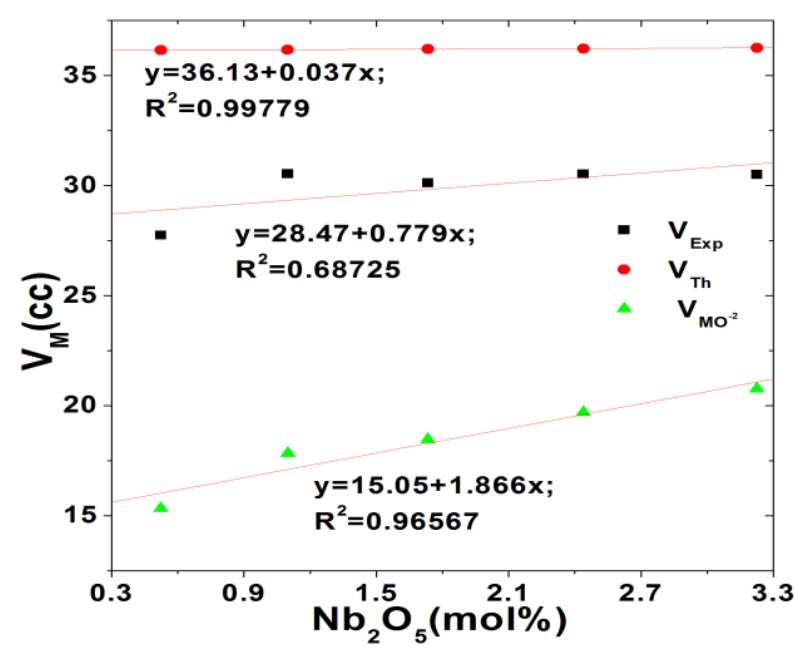

Fig.3 Molar volume and Oxygen molar volume vs. mol\% concentration of Niobium doped BGO1:3 glasses. Lines are linear fit to the data. $\mathrm{R}^{2}$ values are measure of goodness of fit

On further substitution the rate of increase in these parameters was lowered. This identifies structural saturation of the system on further replacement.Fig.3 shows that theoretically a rate of $3.7 \%$ increase is expected in molar volume with $x \%$ increase in niobium however the experimentally observed rate is $77.9 \%$ increase while the oxygen molar volume depicted fast increase at the rate of $186.6 \%$. This can be understood as each Niobium ion donates 2.5 oxygen atoms in the network as compared to bismuth and germanium which can collectively provide 2.5 oxygen atoms if in ratio 1:3. Number of oxygen atoms is the main contributing factor. It can be assumed that $\mathrm{Nb}^{+5}$ is replacing $\mathrm{Ge}^{+4}$ and modifying the network introducing non-bridging oxygens resulting in extraneous oxygen molar volume. 


\subsection{Thermal Parameters}

\subsubsection{DTA- Curves}

In Table2 are included the characteristic transition temperatures, the parameters derived from transformation temperatures and some optical parameters for the system. The glass transition temperature $T_{g}$ was between $456{ }^{\circ} \mathrm{C}-469{ }^{\circ} \mathrm{C} \pm 2{ }^{\circ} \mathrm{C}$ and onset of crystallization temperature $T_{x}$ varied $516{ }^{\circ} \mathrm{C}-537{ }^{\circ} \mathrm{C} \pm 2{ }^{\circ} \mathrm{C}$ due to a broad exothermic shoulder. Thermal stability factor $\Delta T=T_{x^{-}} T_{g}$ had values $51^{\circ} \mathrm{C} \pm 44^{\circ} \mathrm{C}-69{ }^{\circ} \mathrm{C} \pm 4{ }^{\circ} \mathrm{C}$ showing that except BGO75-Nb10 all other compositions possess comparable stability against devitrification. First crystallization peak apex $T_{p l}$ refers to maximum rate of crystallization for the first crystalline phase that would grow to maturity if temperature is raised above $T_{g}$ and kept in neighborhood of $T_{p l}$. Value of $T_{p l}$ shifted from $589{ }^{\circ} \mathrm{C}-624{ }^{\circ} \mathrm{C} \pm 2{ }^{\circ} \mathrm{C}$. It is the main phase that would crystallize if allowed as observed from relative area under the peak. Crystalline peak $T_{p 2}$ corresponding to some second crystalline phase was observed in all DTA curves except that for BGO1:3-Nb5 and its temperature decreased non-monotonically from $722{ }^{\circ} \mathrm{C}-694{ }^{\circ} \mathrm{C} \pm 2^{\circ} \mathrm{C}$ with increasing niobium doping. Corresponding fraction crystallized from the composition was less as compared to $T_{\mathrm{p} 1}$ phase as depicted by relative areas under peaks. Third crystallization peak was observed for all except BGO1:3-Nb10 around $763{ }^{\circ} \mathrm{C} \pm 2{ }^{\circ} \mathrm{C}$ as shown in Fig.4a. Another crystalline feature centered about $784{ }^{\circ} \mathrm{C} \pm 2^{\circ} \mathrm{C}$ was observed in $1^{\text {st }}$ two glasses. These features in DTA curves indicate the existence of variety of environments and structural regroupings in the reported glasses. These observations are again witnessing different cation coordination states with varying composition by injecting more niobium. None of the glasses in this study melted in the temperature range scanned. From Fig.4b it can be seen that for the psudobinary glass system reported both $T_{g}$ and $\alpha$ varied unanimously. The decrease in $T_{g}$ can be explained as due to modifying character of $\mathrm{Nb}^{5+}$ as it caused oxygen molar volume to increase while $\alpha$ decreased as the density increased due to structural rearrangements caused by coordination number changes [9]. Both differentials $T_{x}-T_{g}$ and $T_{P I}-T_{g}$ exhibited similar response to compositional increase in $\mathrm{Nb}_{2} \mathrm{O}_{5}$. This may imply that $\mathrm{Nb}$ doping pinned nucleation of BGO crystal phase around $T_{P 1}$ in $\mathrm{BGO} 75$ compositions which correspond to $\mathrm{Bi}_{4} \mathrm{Ge}_{3} \mathrm{O}_{12}$ (BGO60) crystallites of stable phase of binary $\mathrm{Bi}_{2} \mathrm{O}_{3}-\mathrm{GeO}_{2}$ system [3].

Table2 Characteristic temperatures $T_{g}, T_{x}, T_{p i} ; \mathrm{i}=1-4, \Delta \mathrm{T}=T_{x}-T_{g}, \Delta \mathrm{T}_{\mathrm{p} 1}=T_{p 1}-T_{g}$, CTE $\alpha$, refractive index $n$, direct allowed band gap energy $E_{g}$ and Urbach energy $U_{E}$ of niobium doped BGO75 glasses

\begin{tabular}{|c|c|c|c|c|c|c|c|c|c|c|c|c|}
\hline $\begin{array}{l}\mathrm{Nb}_{2} \mathrm{O}_{5} \\
\mathrm{~mol} \%\end{array}$ & $\begin{array}{c}T_{g} \\
\pm 2^{\circ} \mathrm{C}\end{array}$ & $\begin{array}{r}\mathbf{T}_{\mathbf{x}} \\
\pm 2^{\circ} \mathrm{C}\end{array}$ & $\begin{array}{r}\mathbf{T}_{\mathrm{p} 1} \\
\pm 2^{\circ} \mathrm{C}\end{array}$ & $\begin{array}{r}\mathbf{T}_{\mathrm{p} 2} \\
\pm 2^{\circ} \mathrm{C} \\
\end{array}$ & $\begin{array}{r}\mathbf{T}_{\mathrm{p} 3} \\
\pm 2^{\circ} \mathbf{C}\end{array}$ & $\begin{array}{r}\mathbf{T}_{\mathbf{p} 4} \\
\pm \mathbf{2}^{\mathbf{0}} \mathbf{C} \\
\end{array}$ & $\begin{array}{l}T_{x}-T_{g} \\
\pm 4^{0} \mathrm{C}\end{array}$ & $\begin{array}{l}T_{p 1}-T_{x} \\
\pm 4^{\circ} \mathrm{C}\end{array}$ & $\begin{array}{c}\alpha^{*} 10^{-6} \\
{ }^{0} C^{-1}\end{array}$ & $\begin{array}{c}n \\
632 \mathrm{~nm}\end{array}$ & $\begin{array}{c}\mathbf{E}_{\mathrm{gopp}} \\
\mathbf{e V}\end{array}$ & $\begin{array}{l}\mathbf{U}_{\mathbf{E}} \\
\mathbf{e V}\end{array}$ \\
\hline 0.52 & 464 & 521 & 600 & & 764 & 784 & 57 & 136 & & 1.52 & 3.244 & 0.16 \\
\hline 1.10 & 468 & 537 & 624 & 722 & & 785 & 69 & 156 & 5.431 & 1.52 & 3.244 & 0.17 \\
\hline 1.73 & 456 & 516 & 589 & 703 & 764 & & 60 & 133 & 5.708 & 1.57 & 2.740 & 1.39 \\
\hline 2.44 & 469 & 520 & 596 & 664 & 762 & & 51 & 127 & 7.333 & 1.62 & 2.701 & 1.47 \\
\hline 3.23 & 460 & 518 & 595 & 719 & 763 & & 58 & 135 & 6.899 & 1.26 & 2.630 & 1.07 \\
\hline
\end{tabular}
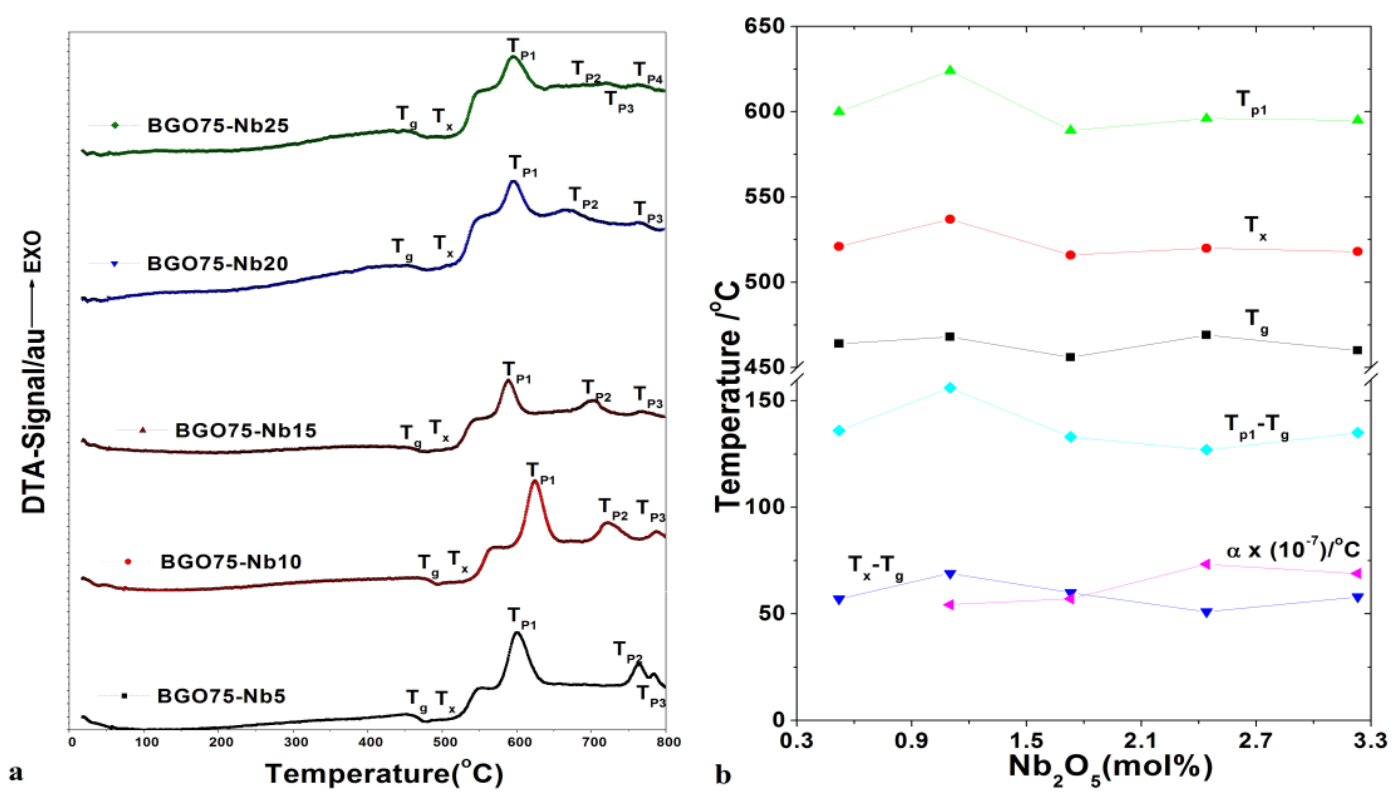

Fig.4a) DTA-curves for Niobium doped BGO75 glasses in temperature range $25-800^{\circ} \mathrm{C}$; b) Variation of characteristic Temperatures $T_{\mathrm{x}}, T_{\mathrm{g}}, T_{\mathrm{P} 1}, T_{\mathrm{x}}-T_{\mathrm{g}}, T_{\mathrm{P} 1}-T_{\mathrm{g}}$ and CTE $(\alpha)$ vs. mol\% concentration of $\mathrm{Nb}_{2} \mathrm{O}_{5}$ for the System 


\subsubsection{Thermal Coefficient of Linear expansion}

The values of thermal coefficient of linear expansion (TCE) $\alpha$ were between $5.431 \pm 0.001 * 10^{-6} \mathrm{~K}^{-1}$ and $7.333 \pm 0.001 * 10^{-6} \mathrm{~K}^{-1}$ as reported in the Table 2 and plotted in Fig.4b. TCE is also a structural probe for solid state.An inverse relation was observed between $\alpha$ and density $\rho_{\text {exp }}$, between $\alpha$ and refractive index $n$ while a direct proportion to the molar volume $V_{M \text { exp }}$ of the composition. This is expected as an open network expands more on heating. $\alpha$ increased from $5.431 * 10^{-6} \mathrm{~K}^{-1}$ to $7.333 * 10^{-6} \mathrm{~K}^{-1}$ as niobium concentration increased from 1.10 to $2.44 \mathrm{~mol} \%$ the maximum value of $\alpha$ and then decreased to $6.899^{*} 10^{-6} \mathrm{~K}^{-1}$ for further increase in niobium concentration to $3.23 \mathrm{~mol} \%$. This may be due to co-ordination number change related to structural rearrangement in the network due to larger radius of $\mathrm{Nb}^{+}$cation.

\subsection{Optical Parameters}

In Table2 are included some optical parameters, the refractive index $n$, direct band gap energy $E_{g}$ and Urbach energy $U_{E}$ of the glass system investigated. Refractive index was calculated by reflectance at $632 \mathrm{~nm}$ using the relation that permittivity $\varepsilon$ is related to refractive index by

$$
\epsilon=n^{2}=\frac{1+\sqrt{R}}{1-\sqrt{R}}
$$

Direct band gap energy $E_{g}$ and Urbach energy $U_{E}$ of the glass system were calculated from the UV-NIR absorbance spectra of the glasses using, Lambert-Beer's law that optical absorptivity $\boldsymbol{\alpha}(\boldsymbol{v})$ of the absorbing medium of thickness $d$ traversed is given by

$$
\alpha(v)=\frac{1}{d} \ln \left(\frac{I}{I_{o}}\right)
$$
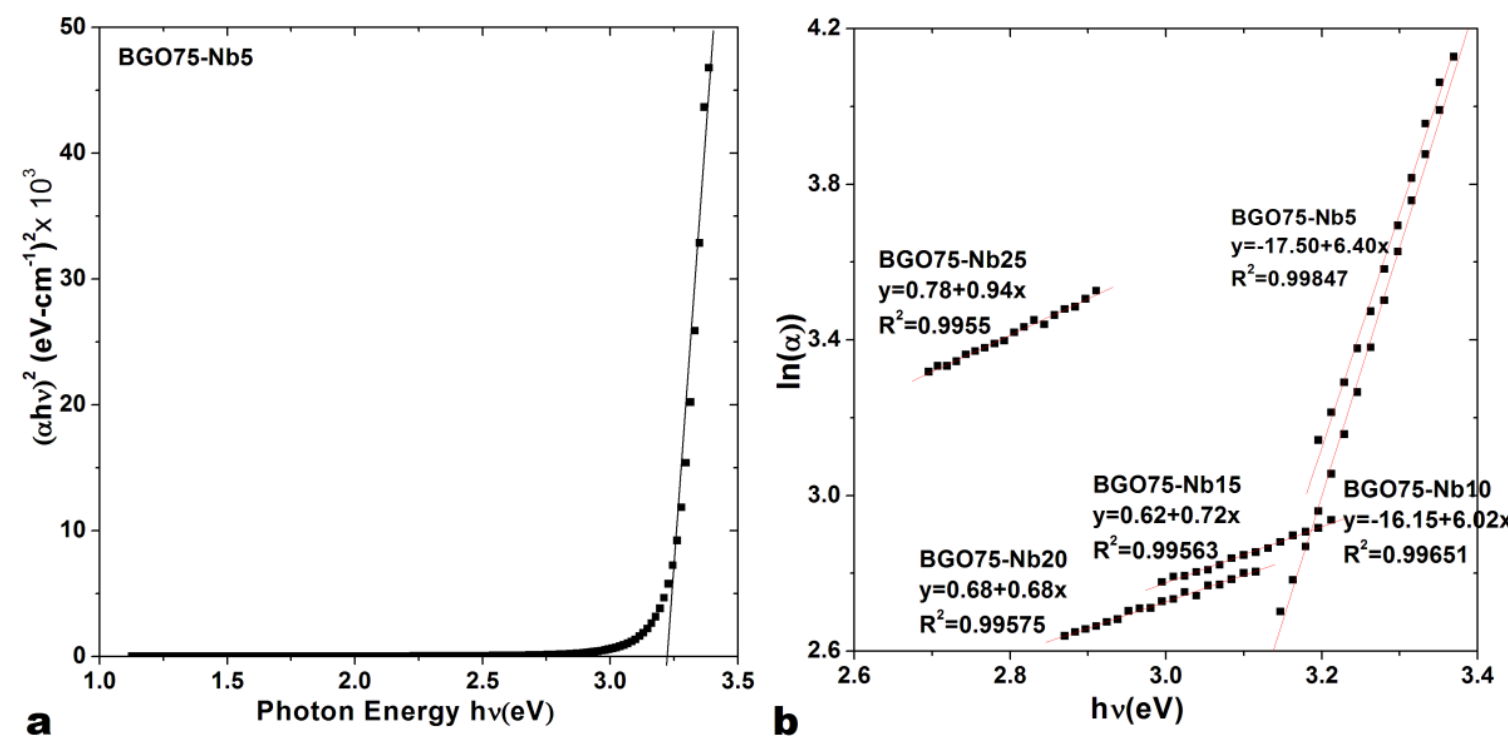

Fig. 5a) $(\boldsymbol{\alpha} \boldsymbol{h} \boldsymbol{v})^{2}$ vs. $\boldsymbol{h} \boldsymbol{v}$ plot for BGO75- Nb5 glass according to Mott and Davis relation for allowed direct band gap energy $\mathrm{E}_{\mathrm{g}}$; b) linear fits to mobility edges according to Urbach Law from Tauc's plot for Niobium doped BGO1:3 glass system

Applying Mott and Davis relation in amorphous solids,

$$
\boldsymbol{\alpha} \boldsymbol{h} \boldsymbol{v}=\boldsymbol{B}\left(\boldsymbol{h} \boldsymbol{v}-\boldsymbol{E}_{\boldsymbol{g}}\right)^{r} ; \boldsymbol{r}=(2,3,1 / 2,1 / 3)
$$

Where 2: indirect allowed, 3: indirect forbidden, $1 / 2$ : direct allowed, $1 / 3$ : direct forbidden excitation of electrons across the mobility gap of the amorphous solid. $h v$ is the energy of the incident photon of frequency $v$. $E_{g}$ corresponds to bandgap energy and B is band tailing parameter that arises due to amorphous nature of glassy medium in contrast to crystals which have sharp absorption edges.

Fig.5a shows $(\alpha h v)^{2}$ vs. $h v$ plots which correspond to $\boldsymbol{r}=1 / 2$ and produced a good fit of the data for determination of mobility gap energy $E_{g}$, reported in Table2 and Fig.6. The plot reveals that niobium doped BGO glasses reported are direct bandgap solids and their excitation occurs across allowed states. Optical bandgap energy $E_{g}$ ranged from 3.244-2.630 eV decreasing with increasing concentration of niobium. This value of band gap is less as compared to simple BGO bandgap energy reported earlier 3.7-3.1 eV by Stentz [12]. 
Urbach energy $U_{E}$ of the glasses was calculated from Tauc's Plot (Fig.5b ln $\alpha$ vs. photon energy) as inverse of the slope of the straight line segment near absorption edge. It extended from $0.16-1.47 \mathrm{eV}$ within the band gap. Urbach energy is the measure of the extension of the density of states function within the bandgap of glass from the total order of related crystalline state in terms of band theory of solids. The glass BGO75-Nb20 exhibited the greatest Urbach energy. It was concluded that the system which is $2 \mathrm{~d}$ ion doped glass obeyed Urbach law as shown in Fig.5b similar to glasses reported earlier by P.Petkova for 3d and 4d ions doped BGO glasses [13].

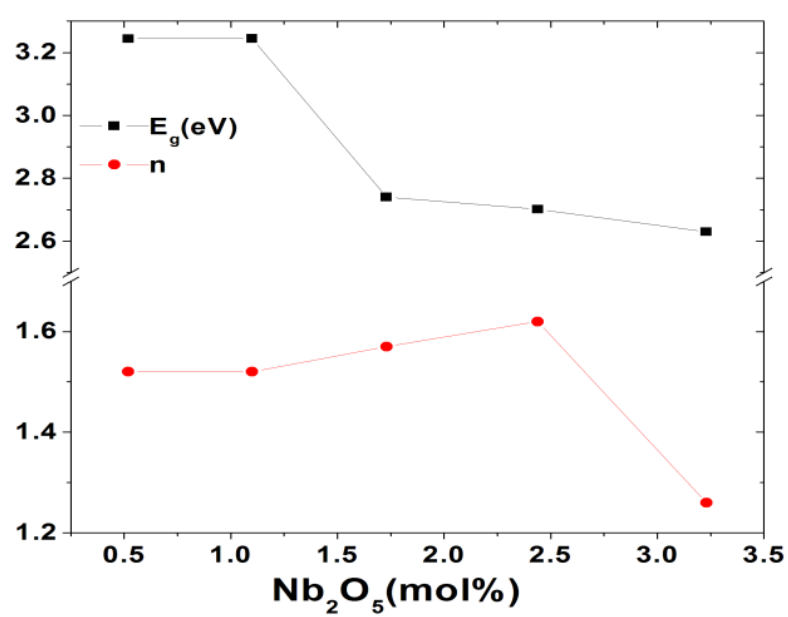

Fig. 6 Variation of Energy band gap $E_{g}$ and refractive index $n$

Fig.6 reveals that both optical band gap energy $E_{g}$ and refractive index $n$ exhibited a plateau against $\mathrm{Nb}$ doping initially similar to $T_{g}$ up to BGO-Nb10. On further doping anion network softened with increase in the molar volume and oxygen molar volume. That may be due to more ionic character of $\mathrm{Nb}^{5+}$ ion causing depolymerization and increased NBOs in the network thus assigning modifying behavior to $\mathrm{Nb}_{2} \mathrm{O}_{5}$. The increase in NBOs caused $T_{g}$ to decrease but due to polarizing strength of $\mathrm{Nb}^{5+}$ replacing $\mathrm{Ge}^{4+}$ ions the refractive index raised continuously up to BGO75-Nb20. On further doping some structural relaxation caused refractive index to decrease. Mobility gap $E_{g}$ (Fig.6) and Urbach edge $U_{E}$ (Fig.5b) also corroborated abrupt change in their values from BGO75-Nb10 to BGO75-Nb15. This may be explained firstly, due to structural co-ordination number change around $\mathrm{Bi}^{3+}$ and/or $\mathrm{Ge}^{4+}$ cation environments resulting in decreased band gap energy.
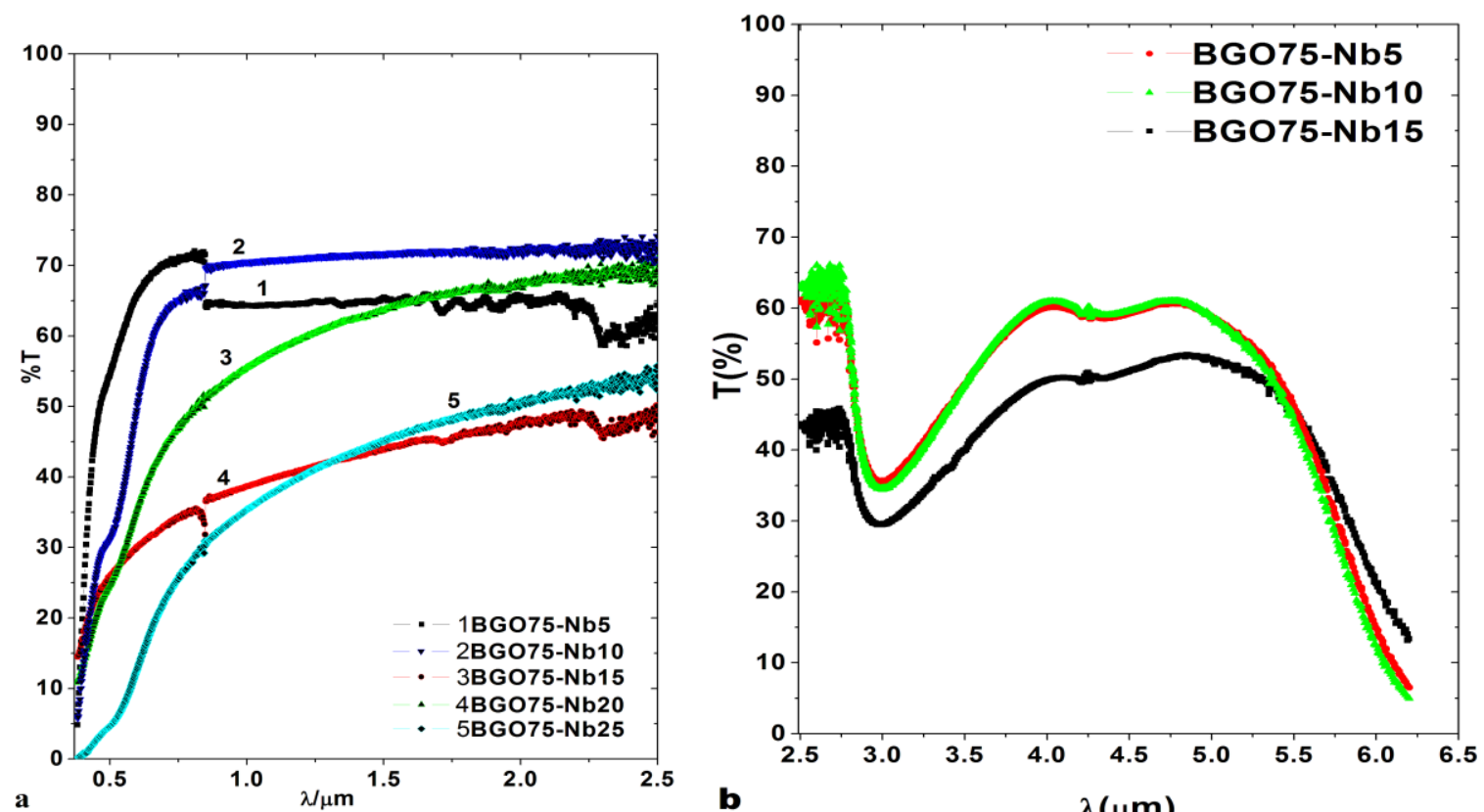

Fig.7a) VIS-NIR transmission spectra of BGO75glasses; b) IR-Transmission spectra of some Niobium doped

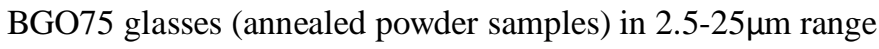


Secondly, extension of density of states function also tailed into the bandgap due to impurity states of niobium. Extended Urbach exponential edge is manifested due to coupling of phonons and excitation of electrons across these impurity states $[8,11]$. The appearance of second and third crystalline features depicted by DTA curves in Fig.2 support this argument.

Fig.7a shows that the optical cut-in wavelength lies at the edge of visible spectrum of electromagnetic radiation for samples of thickness $1-2 \mathrm{~mm}$. \% T decreased as thickness of the sample disk increased. Niobium concentration also affected cut in wavelength. It was red shifted as the niobium concentration increased which agrees with the decrease in bandgap energy and increase in Urbach energy that is function of structural arrangement and impurity states [13].

In Fig.7b are reproduced the IR transmission spectra of $\mathrm{BGO} 75$ powder samples pre-annealed at $350^{\circ} \mathrm{C}$ for one hour. Cut off wavelength is extended into MIR region up to $6.4 \mu \mathrm{m}$. From Fig.7b it is evidenced that IR spectra of BGO glasses are sensitive to compositional variance. The glass BGO75-Nb10 exhibited least absorption and constant percent transmission up to $71 \%$ for disk samples across $0.60 \mu \mathrm{m}-2.5 \mu \mathrm{m}$ in VIS-NIR region. This transmission efficiency is greater than binary BGO60 glasses [7]. Structural investigation of the lattice vibrations in glass system around cation environment can be performed through IR absorption along with complementary micro Raman spectra in the fingerprint range of $400 \mathrm{~cm}^{-1}-1300 \mathrm{~cm}^{-1}$ which is the subject of next investigation. IR transmission in un-annealed powder samples was larger than annealed powders. This may be explained as effect of structural relaxation and more local order in the glasses. The main molecular vibrational absorption in powder samples was corroborated only in longer wavelength region around $1300 \mathrm{~cm}^{-1}$ to $400 \mathrm{~cm}^{-1}$ range.

\section{Conclusions}

In ternary glass system BGO75-Nb transparent glasses were successfully molded through normal meltquench technique. Investigated physical, thermal and optical parameters showed that Niobium initially modifies BGO75 glass system and then imparts structural changes in the glass with increasing mol\% concentration of $\mathrm{Nb}_{2} \mathrm{O}_{5}$. This was revealed by a plateau in its physico-optical and thermal parameters. The value of CTE of glass system was low implying mechanical strength of the niobium doped BGO system. They withstood good thermal and mechanical stability. Optical parameters $n, E_{g}$ and $U_{E}$ were affected by both NBOs and polarizing strength of the Niobium cation and therefore varied non-monotonically. The glasses obeyed Urbach Law. The origin of Urbach tails was assigned to impurities/defect states in the bandgap and excitations coupled with lattice phonons. The MIR transmission window was extended to $6.4 \mu \mathrm{m}$.

\section{Acknowledgements}

Authors wish to pay their gratitude to Higher Education Commission, Pakistan for providing financial support for conducting above research. PIN \# 063-161105-PS3-277.

\section{References}

[1] M. Abdel-Baki and F. El-Diasty, Optical properties of oxide glasses containing transition metals: Case of titanium- and chromiumcontaining glasses, Current Opinion in Solid State Material Science, 10, 2006, 217-222.

[2] S. Polosan, E. Apostol, M. Secu and G.H. Aldica, BGO glasses: structural and optical characterization, Physical status solidi C 2(1), 2005, 93-96.

[3] L. Dimesso, G. Gnappi, A. Montenero, P. Fabeni and G.P. Pazzi, Crystallization behavior of Bismuth Germanate glasses,. Journal of Material Science 26, 1991, 4215-4219.

[4] V.P. Zhereb and V.M. Skorikov, Metastable states in bismuth-containing oxide systems, Inorganic Materials 39(2), 2003, $121-145$.

[5] S. Polosan. Characterization of BGO glass-ceramic materials, Journal of Non-Crystalline Solids 355, 2009, 1900-1903

[6] S. Polosan, F. Nastase and M. Secu, Structural changes during the crystallization of the $\mathrm{Bi}_{4} \mathrm{Ge}_{3} \mathrm{O}_{12}$ glasses, Journal of Non-Crystalline Solids 357, 2011, 1110-1113

[7] M.A. Villegas and JMF Navarro, Physical and structural properties of glasses in the $\mathrm{TeO}_{2}-\mathrm{TiO}_{2}-\mathrm{Nb}_{2} \mathrm{O}_{5}$ system, Journal of European Ceramic Society 27, 2007, 2715-2723.

[8] S. Polosan, Dynamics of energy absorption versus crystallization in Bi4Ge3O12 (BGO) amorphous materials, Material Research Bulletin 45, 2010, 1492-1495

[9] Khair-u-Nis and M.A. Chaudhry, Non-isothermal crystallization kinetics of niobium-doped BGO70 glasses, Journal of Material Science 48, 2013, 1368-1376

[10] V. Dimitrov and T. Komatsu, Classification of Simple Oxides: A Polarizability Approach, Journal of Solid State Chemistry 163, 2002, $100-112$.

[11] V. Dimitrov and T. Komatsu, An Interpretation of Optical Properties of Oxides and Oxide Glasses in Terms of the Electronic Ion Polarizability and Average Single Bond Strength (Review), Journal of University of Chemical Technology and Metallurgy 45(3), 2010, 219-250

[12] D. Stentz, H.B. George, S.E. Feller and M. Affatigato, Comparison of the optical cutoffs of bismuth borate with bismuth germanate glasses, Physics and Chemistry of Glasses European Journal of Glass Science and TechnologyB 41(6), 2000, 406-408. ingentaconnect.com/content/sgt/pcg/2000/00000041/00000006/4106406

[13] P. Petkova, Urbach's rule of Bi4Ge3O12 doped with 3d and 4d ions, 2011, Optical Materials 34,265-268. 